The Science and Engineering Postdoc Experience through the Lens of Liminality

Irene B. Mena, University of Illinois, Urbana-Champaign

Irene B. Mena has a B.S. and M.S. in industrial engineering, and a Ph.D. in engineering education. Her research interests include first-year engineering and graduate student professional development. 


\title{
The Science and Engineering Postdoc Experience through the Lens of Liminality
}

\begin{abstract}
The purpose of this study is to learn about the experiences of postdoctoral scholars (postdocs) in science and engineering fields. It is guided by the theory of liminality. Forty-two postdocs employed at a Mid-Atlantic, research-intensive University, participated in individual interviews to describe their experiences as postdocs at the University. This paper provides answers to the following three research questions: (1) What are the benefits and challenges of science and engineering postdoc positions? (2) What liminal experiences characterize the postdoc position? and (3) What recommendations to improve the postdoc experience emerge from this study?
\end{abstract}

\section{Introduction}

The purpose of this study is to learn about the experiences of postdoctoral scholars (postdocs) in science and engineering fields. A postdoc is typically defined as an individual who has recently obtained a doctoral degree, and is completing further training before moving on to a more permanent position, such as that of a faculty member.

This study was guided by the theory of liminality. Liminality, a theory from the field of anthropology, refers to a stage of transition. Individuals in a liminal state are defined as being in a somewhat unclear state that is between two clearly defined states. The postdoctoral experience, being between the graduate student and faculty states, can be considered a liminal state.

Forty-two postdocs (19 in engineering and 23 in science) employed at a Mid-Atlantic, researchintensive University, participated in individual interviews to describe their experiences as postdocs at the University. The reasons for conducting this study were two-fold, and provide the framework for the structure of this paper. First, the study sought to better understand the science and engineering postdoc experience at the University. As such, the current paper outlines the typical responsibilities associated with the position, as described by the interview participants, and provides answers to the first research question: (1) What are the benefits and challenges of science and engineering postdoc positions?

Second, the study sought to view the postdoc experience through the lens of liminality, with the goal of using insights from liminality to provide recommendations to improve the postdoc experience. Thus, the final two research questions are: (2) What liminal experiences characterize the postdoc position? and (3) What recommendations to improve the postdoc experience emerge from this study?

While other studies have looked at the postodoc experience, this study is unique in two main ways. First, it focuses only on science and engineering postdocs. Second, the data is analyzed and interpreted through the lens of liminality, a theory that has not been commonly used to explain the postdoctoral experience. In addition to being useful to postdocs themselves, findings 
from this study will be useful to faculty, departments, and universities that employ postdocs. Findings will also be useful to current doctoral students and faculty who advise doctoral students, as these students consider next steps in their careers.

\section{Background literature}

Postdoctoral scholars, or postdocs, are usually considered to be individuals who have recently finished their doctoral degrees, but are not yet in a permanent position. The Association of American Universities (AAU) Committee on Postdoctoral Education ${ }^{1}$ provided several criteria to define a postdoc appointment. These criteria include: "the appointee was recently awarded a $\mathrm{Ph.D.} \mathrm{or} \mathrm{equivalent} \mathrm{doctorate...} \mathrm{in} \mathrm{an} \mathrm{appropriate} \mathrm{field[,]...} \mathrm{the} \mathrm{appointment} \mathrm{is} \mathrm{temporary[,]...} \mathrm{[it]}$ involves substantially full-time research or scholarship[,]... [and it] is viewed as preparatory for a full-time academic and/or research career," among others. Although this is what is typically thought of regarding postdocs, a consistent definition of what a postdoc position actually is, is still lacking, which makes studying the postdoc experience a challenge ${ }^{2}$.

Possibly due to the lack of a consistent definition of what a postdoc appointment is, there can be many different responsibilities that characterize postdoc positions. Akerlind ${ }^{3}$ writes that there is "substantial variation within postdoc roles and responsibilities, even within the same disciplinary area." These responsibilities, as listed by Akerlind ${ }^{3}$ include: being completely in charge of a research project and all it involves; being a "project manager" who has certain "independence and flexibility" with a research project, though only within the already-established goals of the project; being a "pair of hands" who works on experiments and analyses already specified by the project and/or supervisor; and being an "aide" who would complete tasks assigned by the supervisor, where these tasks may not necessarily be related to any specific research project (for example, supervising students). Regardless of the responsibilities that come with their positions, postdocs play an important role in research: they are responsible for the "sometimes exhilarating, sometimes tedious day-to-day work" in research projects ${ }^{4}$ and are also responsible for a "disproportionate share of new discoveries".

There are many benefits and opportunities associated with a postdoc position. The main ones are that this is a time for the postdocs to improve their "scientific and technical abilities," to "demonstrate originality, creativity, and productivity," and to publish papers ${ }^{4}$. Postdoc experiences also provide postdocs with more independence and self-direction, and with opportunities to work with advisors who will ideally help them become better researchers and from whose professional experiences postdocs will learn ${ }^{4}$. In addition, the postdoc experience provides postdocs with time to network at professional meetings ${ }^{6}$. These benefits are expected to be helpful to them in the process of later obtaining permanent positions. There are benefits even after they are in their permanent positions; for example, those postdocs who continue to faculty careers can expect benefits such as more "contact and collaboration with peers from other countries" and "higher scientific outputs than their colleagues"7.

There are also some negative aspects associated with a postdoc position. First, for some postdocs, the experience fails to provide some of the necessary requirements of a successful postdoc experience, such as opportunities for "growth toward independence," guidance, and mentoring relationships ${ }^{4}$. Also, in many cases, advisors and institutions do not give postdocs the 
"status, recognition, and compensation that are commensurate with their skills and contributions to research"4. In addition, there is a lack of explicit standards, which means that working conditions and salaries can vary even within one department ${ }^{5}$. Finally, the "institutional status" of postdocs remains "poorly defined"4. Because they are considered neither students nor faculty/staff, they may "receive the benefits and protections of neither".

The length of time of a postdoc experience, described as being a period of between three to five years $^{6}$, can vary for each postdoc. According to the National Academy of Sciences (NAS), the National Academy of Engineering (NAE), and the Institute of Medicine (IOM) ${ }^{4}$, the median time individuals spend as postdocs is 3.5 years, but according to the National Science Foundation (NSF) Survey of Doctorate Recipients, the median time for science, engineering, and health postdocs is approximately two years ${ }^{8}$. These lengths of time can vary depending on whether individuals have a temporary U.S. visa, are married, and have dependents ${ }^{9}$. Some concerns have been raised regarding the length of a postdoc experience, because being a postdoc for longer than what is considered the norm may negatively impact an individual's job opportunities. Being a postdoc for too long may cause potential employers to question the individual's "capabilities, goals, and motivations" and may even result in being rejected ${ }^{10}$. This may be one of the factors that has motivated some institutions to place a five-year limit on the amount of time an individual can be a postdoc ${ }^{11}$.

In various fields, a postdoc experience has become a necessary step before a permanent position $^{4}$, especially within academia ${ }^{10}$. One of the reasons why postdoc positions have become more common is the "increasing unbalance between the number of available tenured and tenuretrack jobs in higher education and the number of doctorates"7. But regardless of the reasons, there has been an increase in the number of doctorate recipients who transition to a postdoc position $^{8}$. According to the 2006 NSF Survey of Doctorate Recipients, $45 \%$ of science, engineering, and health doctorate recipients continued on to a postdoc position ${ }^{8}-$ that is, a considerable proportion of individuals with science and engineering doctorates become postdocs. Therefore, it is important to look at the postdoc experience in terms of what postdocs learn and accomplish, but also what challenges and needs they face, with the goal of making the necessary changes to improve the experience. As stated by Scaffidi and Berman ${ }^{12}$, "[t]he academic literature on the postdoctoral experience is sparse." This paper seeks to contribute to the academic literature in this area by describing and discussing the postdoctoral experience, based on the participants' descriptions, and by providing suggestions to improve this experience. While this study is set in the context of science and engineering at a mid-Atlantic research University, it is expected that the resulting recommendations will benefit postdocs in all fields of study.

\section{Research Questions}

The purpose of this study is to learn about the experiences of postdocs in science and engineering fields. It answers the following research questions: (1) What are the benefits and challenges of science and engineering postdoc positions? (2) What liminal experiences characterize the postdoc position? and (3) What recommendations to improve the postdoc experience emerge from this study? 


\section{Theoretical Framework}

This study is guided by the theory of liminality, originally from the field of anthropology. The anthropologist Arnold van Gennep studied the rituals characteristic of different life periods, what he called rites of passage. According to van Gennep ${ }^{13}$, every time an individual changes from one situation or state of being to the next, this change consists of three stages: separation, transition, and incorporation. In the separation stage, the individual leaves a state of being, and in the incorporation stage, the individual has completely embraced or joined a new state of being. The middle stage, the stage of transition, is called liminality ${ }^{14}$. An individual in this stage of transition can be referred to as the "transitional-being" or the "liminal persona" personae are invisible in the sense that they are "at once no longer classified and not yet classified"15. They are "neither-this-nor-that, here-nor-there, one-thing-not-the-other"14; they are "structurally if not physically invisible" ${ }^{\text {. }}$. Liminal personae also experience a paradox: they are "both this and that"14.

Individuals in the liminal state experience ambiguity, because the characteristics of this state are not those of the state they just left or the state they are going towards ${ }^{14}$. While rites of passage generally describe life events such as birth and marriage, Turner ${ }^{14}$ explains that they also relate to "entry into a new achieved status," such as political office or membership into an exclusive group.

The rites of passage can be used to describe the postdoctoral experience. Postdocs have gone through the stage of separation: they have left the graduate student state and all the responsibilities and rights that come with this state. They will at some point be incorporated into a new state - often in academia, but not necessarily. In the meantime, they are between the two, in the liminal state.

\section{Data Gathering and Analysis Methods}

Data were collected via individual interviews with 42 postdocs employed at a large, researchintensive, mid-Atlantic University. To contact these postdocs, an email list was obtained from the University. This list contained the contact information for the 249 individuals the University considered postdocs in science and engineering fields. Of the 42 postdocs who agreed to participate in the interviews, 19 (18 male, 1 female) were from the College of Engineering, and 23 (18 male, 5 female) were from the College of Science. The interviews followed a semistructured protocol that asked the postdocs to talk about their responsibilities and the experiences that characterized their postdoc positions. These interviews were recorded and then transcribed for analysis.

The first step in the data analysis process was to read all interviews with the purpose of getting a "general sense" of the data and the main ideas shared by the participants ${ }^{17}$. Codes, or labels assigned to "a piece of text that contains an idea or information"18, were used to identify the data. No predetermined codes were used; all codes emerged from the data. This coding process took place within the NVivo software. The codes were used first to determine themes that characterized each participant's experience and then to determine themes that characterized the postdoc experience as a whole. The final step in the data analysis process is interpretation ${ }^{17}$. 
The data were interpreted using the literature on liminality and the literature on postdoc positions.

Two main steps were taken to ensure the trustworthiness of the data and results. First, to remain alert to personal biases as a researcher, the questions suggested by Glesne ${ }^{19}$ were considered throughout the data analysis as well as throughout the interpretation processes: "Whom do I not see? Whom have I seen less often? Where do I not go? Where have I gone less often?" Second, as suggested by Glesne ${ }^{19}$, the data were shared with fellow researchers and colleagues as a means to ensure trustworthiness.

\section{Results}

Of the 42 posdocs in this study, $69 \%$ were in their first postdoc position, $29 \%$ were in their second, and $2 \%$ were in their third. Of these $42,48 \%$ were interested in academic positions after their current position, while $29 \%$ were interested in industry. The remaining postdocs either were uncertain of their plans or had plans unrelated to industry or academia. The following sections summarize the reasons these postdocs decided to pursue postdoc positions, and the responsibilities, benefits, and challenges that came with the position. The participants' gender and field are represented by M (male), F (female), SCI (science), and ENG (engineering).

\section{$\underline{\text { Reasons for Pursuing Postdoc Positions }}$}

The postdocs in this study shared the various reasons why they decided to pursue a postdoc position. The first reason for pursuing a postdoc position is interest in doing research. This is how one postdoc described it:

"I enjoy research, and I enjoyed it in my Ph.D. program, and I wanted to carry on some of the work I was doing...And so that's why I chose to do a postdoc. I wanted to carry on some of my work." (F-SCI)

A postdoc position was also seen as a way to get experience and training in certain topics or skills, which is one of the reasons for undertaking these positions. Two postdocs said:

“...after the Ph.D...., I still feel my skills and knowledge in my field [are] not good enough... I needed to find a place to improve myself." (M-SCI)

Another reason why postdocs took on their positions is that they believed it was a necessary step before applying for faculty positions. For example:

“...the Ph.D. holder... at some point will have to go to [a] postdoc to prove $[$ him/herself]... and to show that you are able to cooperate with other teams. So you can say it's a necessary entry in your scientific resume. So yeah, it was my main motivation." (M-ENG)

"It's not that I really like to be a postdoc; it's just I do need some experience before I can get a faculty position... in my field, on average, people have to do at least two or three postdocs before they can get to a faculty position." (M-SCI) 
Finally, for some postdocs, it was the opportunity to get more publications that motivated them to pursue postdoc positions.

\section{$\underline{\text { Postdoc Responsibilities }}$}

Because of the lack of a consistent definition of a postdoc position ${ }^{2}$, it is important to learn about these postdocs' responsibilities, in order to better understand what "postdoc position" refers to in this specific context. The postdocs in this study described the responsibilities that came with their positions, and the main ones were conducting research and helping and occasionally mentoring students. In addition to these two main responsibilities, some postdocs were also in charge of managing a lab and/or equipment, and a minority was required to help their advisors with a class, such as by lecturing when the advisor was unable to do so.

\section{Benefits of a Postdoc Position}

The postdocs in this study talked about the different benefits they associate with their positions. These include: learning and/or improving different skills, being able to focus on research and publications, independence and freedom, and having the opportunity to build their network. Regarding the skills postdocs learn and/or improve, they discussed both research-related skills and professional skills. When describing research-related skills, they specifically talked about learning more about their field, learning new methods, and learning to use new instruments. Some postdocs were in a completely new field, so they had to learn about that field and about the techniques used in that field. For example:

"...I learned several techniques that were instrumental techniques, ... analysis techniques..." (M-ENG)

"I changed fields when I started my postdoc. So I've had to learn a completely new set of experimental techniques and laboratory skills and those kinds of things that I didn't have before." (M-SCI)

When describing the professional skills they learned, they mentioned improving their communication and writing skills, and teamwork/collaboration skills, as well as their abilities to mentor and work with students.

Another benefit of a postdoc position was being able to focus on research and publications, due to the absence of additional responsibilities such as teaching and/or taking classes:

"So here I just do the research. I can focus on...plain, pure science. I am not distracted by classes." (M-ENG)

“...you can completely focus on research, and then you don't have to worry about funding [or] teaching. You can just spend all your days thinking about the research problem...I really like being [a] postdoc because it's very, very helpful in terms of working on hard problems." (M-SCI) 
The postdocs mentioned independence and freedom as additional benefits of postdoc positions. When discussing these benefits, they sometimes compared their experiences as graduate students to their current experiences as postdocs, and described how in their current positions, they have more freedom and independence, but also more responsibility. They said:

“...I think you have more freedom...because as a student, you have to consider whether this work could contribute to a graduation thesis. But as a [postdoc], you are free to think about what you are really interested, what kind of project you want to participate in." (M-SCI)

"I think I'm more independent. Ph.D. means you have to...be under your [advisor]. So postdoc means you are given a project. Whatever you want to do, you can do...I'm more free, but I'm more responsible, too." (M-SCI)

One final benefit of postdoc positions is that they provide the opportunity to build the postdocs' network. Two ways this occurred was either through conferences or through the postdocs' advisors, as described below:

“...I participated in three or four conferences this year, and from that, I can communicate with a broader community..., and so... you know more people..." (M-SCI)

"I've [made] a lot of good personal connections, good networking opportunities. ...when visiting speakers come in, my boss will say, 'Oh, you need to meet soand-so. I know him, and he's great.' And I meet that person and they are great. I wouldn't necessarily have done that without my mentor providing the opportunities for me. ...it's been very positive." (M-SCI)

\section{Challenges of a Postdoc Position}

There are different challenges that come with a postdoc position. The main one is the level of stress, due to the many responsibilities and pressure to publish, that characterizes the position. For example:

"It's a very high-stress job... and you have to put in extra hours because you're in a scientific field, and there is always pressure and deadlines for budgets...Sometimes - most of the weekends, I work." (M-ENG)

"I think for [science] students, the [hardest part] is the publication. So if you want to find a good job... you have to keep publishing. This is a most challenging part." (M-SCI)

In addition to the amount of responsibilities, stress is sometimes the result of having to learn new topics and methods in a short period of time, as described by this postdoc: 
“...challenges mostly lie [when] you are expected to perform on day one even though you may not have any background in the research the lab is doing, but you have to pick up those skills very quickly, and you shouldn't expect to be doing anything else in your personal life or professional life for the first few months until you get those skills up and running." (M-ENG)

Relationships with advisors were also sometimes challenging for postdocs, although the reasons why those relationships were challenging varied. For some postdocs, communicating with their advisors was challenging, and they had to learn to adapt to their supervisors' ways of communicating:

"...it can be challenging sometimes to figure out exactly what my boss wants or if [he/she] really means what [he/she] says." (M-SCI)

"It's a challenge with my new bosses being different in the way of communication and everything to my old boss. I got used to my old boss. I knew how to take him. And the new one, I don't so far... I am more confused and irritated by how [he/she] reacts sometimes. And it's a challenge, but also it's learning." (F-SCI)

For others, challenges with their advisors were a result of the differences between their expectations and their advisors' expectations:

"I think just conflict of personalities or expectations and things of that nature. So that has been probably slightly negative... with [my advisor]. We get on on a personal level, but on the professional level, we butt heads a little bit." (M-SCI)

"Negative could be that I thought [as] a postdoc... you would be...treated [more] like an individual scientist and less like a student. So I felt [I was too] supervised considering that I'm not a student, but I'm a postdoc. ... So I didn't feel treated like a colleague; I feel treated more like a student." (M-ENG)

The advisors' availability was sometimes an additional challenge:

"Negative experience may be...the interactions between me and my advisor [are] probably...too few... So I can do my work in most cases by myself independently. But sometimes, for example, when I write a paper if I want to publish,...I have to let my advisor read it and approve it. But if he is busy, then the process will become very slow. And also..., if possible,... I hope [my] advisor can get involved in my studies, not in [a very detailed way] but... some important steps should be discussed together...So in one word, I think... more interactions with the advisor may be necessary." (M-SCI)

\section{Liminal Experiences and Resulting Challenges}

In addition to the already-stated challenges, other challenges postdocs faced were due to the liminal experiences associated with the position. Postdocs found it challenging to be in a 
position that was not stable or permanent. One postdoc described the position as being a "temporary stage," and another as an "intermediate status." Different postdocs described different liminal experiences and associated challenges. These are summarized below:

1. The postdoc position is a short-term position, which leads to postdocs feeling worried and anxious about finding a permanent job:

"Sometimes..., many postdocs feel like their position is not stable.

Sometimes, they feel the stress about getting better job soon... that is the negative thing." (M-ENG)

"...I want to get some good publications, and possibly two years or three years later, I will try to find a more stable position. Since [the postdoc position] is, I feel - actually, I feel not very comfortable about it. I feel just not very stable - just a little bit anxious about my future.” (M-ENG)

2. They struggle with determining the appropriate amount of time they should spend as postdocs, both in terms of (a) spending a sufficient amount of time, and (b) not spending too much time as postdocs. Regarding (a), while they are interested in moving on to a permanent position, they realize that in order to do so, they have to be successful in their postdoc positions, which sometimes means staying longer as a postdoc:

"The time period that you're there... Technically, my postdoc could have ended [this year] because it was a year with the option of the second year. So I could have left. But the concept of being somewhere for a year just seemed absolutely ridiculous to me. It's just not a long enough period of time to warrant doing anything. But at the same time, it's like, 'Okay, this is another three years before I can start my real life or my real job or whatever.' So it's really hard." (F-SCI)

Regarding (b), although they feel they should be in a postdoc position long enough to get the training and publications they desire, they also have to think about not spending too many years as a postdoc, or they risk becoming what one postdoc described as "damaged goods" ("if you postdoc too long, you're damaged goods"). Sometimes it is time to move on:

"I would like to move on. I think being [a] postdoc for more than three years gets on your nerves sometimes. I like to be [a] postdoc, and I like to work as a researcher. But at some point, you feel like you can do more than just a postdoc. You can teach... advise ... write grant proposals independently. And so that's what I want to change - but not within [the] postdoc but moving on to the next position." (M-ENG)

"I'm quite happy that I did two postdocs, but then I feel now that it's enough essentially because then at some point also, I don't want to be a perpetual postdoc... you are not going to get tenure if you are getting too old." (M-SCI) 
3. Postdocs are not always recognized or provided with support by the University:

“.... lot of university policies or forums don't acknowledge that there is such a thing as a postdoc, right?...You'll get some survey, and it says, 'Well are you student, staff, faculty, or grad student?' It's like, 'No. I'm none of the above.' So I guess that's a minor challenge.” (M-SCI)

“...sometimes it's hard to fit into the campus community because there's all these support structures for faculty or staff or students, but postdocs there's so few of us that we're lost in the mix." (M-SCI)

4. Postdocs sometimes struggle with not knowing and not being known by members of their community:

"...to the rest of the department, we kind of are transient members... very few people know who we are because we don't take courses... so we don't have the profile that graduate students would have. And so a lot of faculty members have no idea who we are because we just walk by them, and we don't know who they are sometimes, too..." (M-SCI)

5. The postdoc position is not always clear to others:

“... when somebody asks you what you are doing and you say a postdoc, then most people will not know what it is. You have to explain to them what it means... ... When you want to apply for a company...I then say that I've been a research associate which sounds better than postdoc which is a bit like - again, they maybe wonder what is that exactly... most people don't know, so I always have the problem of explaining what exactly I do. ....If you say you're a graduate student, everybody will understand... Well, as a postdoc you are not working for a degree or anything. You do research, but it's just a more - you get less pay and you don't have much security." (M-SCI)

\section{Discussion}

In this study, participants shared the experiences that characterized their postdoc positions. Many of the experiences, benefits, and challenges that arise from a postdoc position are characteristic of the liminal state and can be considered liminal experiences. As explained in a previous section, postdocs are in a liminal state: they are no longer graduate students, but are not yet faculty or in any other permanent position. The postdoc could therefore be described as being "neither what she or he was nor what she or he will become" ${ }^{20}$. The postdocs in this study recognize that they are in a liminal state, though they did not use the same terms. The liminality of the postdoc position is best described by one of the postdocs:

"But the downside of being a postdoc are all of these other issues that you're somewhere between a graduate student and somewhere between being an employee, not just at [the University] but in general, this transient species of - 
sometimes your advisor teaches you, [sometimes] you're in an advisory role; you're working with graduate students and helping them, and other times, you feel very much like a graduate student or an undergrad. So this is [a] weird mixture of things." (F-SCI)

The postdocs shared some of the reasons why they decided to pursue a postdoc position. These reasons include an interest in doing research, a way to get experience and training in certain topics or skills, and the opportunity to get more publications. They also mentioned that having a postdoc was required in their field if they wanted to get certain positions afterwards. In certain fields, according to NAS et al. ${ }^{4}$, having one or more postdoc positions has in fact become a requirement to obtain a position in industry or academia. Similarly, the AAU Report ${ }^{1}$ states that in approximately two-thirds of the departments in their study, all newly hired professors had postdoc experience, and that in certain fields, "a postdoctoral appointment has become the de facto terminal academic credential." In this sense, postdocs can be considered "ritual liminars": they are on their way to a higher status, but in the meantime are without a clear status, as part of the "ritual" required by the culture ${ }^{16}$, in this case, the academic/industry culture, which can sometimes require a postdoc position as a rite of passage.

One of the reasons a postdoc position has become a requirement, or a rite of passage, in many fields is that "graduate school programs cannot alone provide the broad range of knowledge and skills required for modern research" needing to learn or improve certain skills. Another reason is that postdocs want to be able to produce quality work that would later help them get a good position ${ }^{4}$. For the postdocs in this study, their quality work would ideally result in publications.

The postdocs in this study described different challenges that came with the postdoc position, including challenges that can be considered a result of the liminal nature of this position. Two of these challenges were worries about finding a permanent position and doubts about how long to stay in the postdoc position. They desired to move on to a permanent position, but knew that in order to get a good position in the future, they needed to produce satisfactory work as postdocs, and this sometimes meant extending their periods as postdocs. On the other hand, they seemed to be aware of an unspoken time limit when it comes to postdoc positions - being postdocs for longer than this unspoken time period could be detrimental to their future careers, so they struggled with determining the right time to move on. Uncertainty can therefore be considered a characteristic of the postdoc experience for many individuals: uncertainty about whether they will be getting a permanent position in the future, and also about when they should ideally be transitioning to these positions.

Two other challenges that resulted from the liminal nature of the postdoc position were lack of support and recognition by the institution, and not knowing or being known by the members of their community. While faculty, staff, and students tend to have clearly-designated policies, responsibilities, and sources of support, this was not perceived by some to be the case for postdocs, which meant that for them, it can be "hard to fit into the campus community" (as described by one of the participants). Something else that contributes to feelings of not fitting in are perceptions of their not knowing or being known by members of the community. Certainly, the postdoc position can be isolating: for example, postdocs don't usually take courses, which limits their interactions with other members of their departments, both graduate students and 
faculty. Limited interactions with other members of their departments lead to a more limited number of relationships, which in turn may lead to feelings of not belonging and even of invisibility.

The two challenges described in the above paragraph illustrate that postdocs are "structurally if not physically invisible in terms of [their] culture's standard definitions and classifications"16, which is a characteristic of being in the liminal state. In university culture, faculty, staff, and graduate students seem to have clear expectations that are assigned to their roles. Postdocs, on the other hand, fall under none of those categories. They have "uncertain status"4 and are "less visible...than students, staff, and faculty" 21 . According to Turner ${ }^{15}$, members of a society tend to see what they expect to see based on the "definitions and classifications of [their] culture." Turner's statement may explain the invisibility experienced by the postdocs in this study: the classifications that are usually expected in higher education tend to be faculty, staff, and graduate student, whereas postdocs "do not easily fit into simple categories at most institutions"4.

While the liminal period is considered to be a "grinding down process," it is also a "rebuilding process" "14: the liminal personae receive verbal and nonverbal instruction and are being "refashioned into specific shapes compatible with their new postliminal duties and rights as incumbents of a new status and state"14. This was true for the postdocs in this study. The benefits they associated with their postdoc positions (learning and/or improving different skills, focusing on research and publications, having independence and freedom, and building their network) are all in different ways preparing them for their future careers, their postliminal states.

As the postdoc position is generally considered a time of professional development and growth, it is not surprising that the postdocs expressed that they learned and improved different skills. What might be somewhat surprising to some who had not considered this other option, is that many postdocs in this study used this experience to learn about and enter a new field, a field different than the ones they obtained their degrees in. The postdoc experience is therefore not only an opportunity to strengthen the skills learned in the doctorate, it can also be an opportunity to enter a field complementary to the individual's doctorate, a chance for an individual to carve out his or her own unique path. This is another characteristic of the liminal state, as the liminal state is a "realm of pure possibility whence novel configurations of ideas and relations may arise" 15 . It can be suggested that the postdocs in this study who were venturing in new fields were doing just that; they were experimenting with their own "novel configurations" of ideas and career possibilities.

In conclusion, the postdoc experience comes with challenges and benefits. For many, it seems to be a period of not belonging and of invisibility. But the postdoc position, being in the liminal state, is also a "storehouse of possibilities,... a striving after new forms and structures" 22 . It is a time to experiment in preparation for the postliminal states, since in liminality, "new ways of acting, new combinations of symbols, are tried out, to be discarded or accepted"14. As a liminal state, the postdoc position can help postdocs be more prepared and confident in their abilities once they transition to their postliminal states. 


\section{Liminality and Its Implications to Improve the Postdoc Experience}

Some recommendations on how to improve the postdoc experience emerged from the study. Specifically, the literature on liminality provides insights on how to improve this experience. In fact, understanding liminality and how it relates to the postdoc position can be beneficial to advisors, institutions, and postdocs themselves. Recommendations for postdocs, advisors, and institutions, based on the interviews and/or supported and explained by the literature on liminality, are below.

First, postdocs should see their positions as the "rebuilding process" 14 that it is. They can use this time to more accurately determine what they would like to do in their postliminal states, and to prepare accordingly. This can be achieved by embracing and learning from the new responsibilities that come with the role, and by seeking out new responsibilities and relationships. This liminal state should also be a time to be proactive, a time for postdocs to "ask for what they need, find their own new resources, meet new people, and solicit invitations to speak about their work" . Postdocs should remember that "developing a proactive mindset hastens the journey from student to professional" ${ }^{\prime 4}$.

Also, while the postdoc experience is a time to network with other professionals and established members of the field, it should also be a time to connect with other postdocs. The liminal state is characterized by the relationship that develops among the liminal personae. Because liminal personae don't have differences in status among themselves, they tend to share an "intense comradeship and egalitarianism" 23 and are "drawn together in bonds of fellowship" 24 . Liminal personae are a "community... of comrades" 15 . Yet, postdocs "sometimes know few or no other postdocs at the institution". As liminal personae, postdocs would benefit from interacting with other postdocs. They can provide each other with support in situations that characterize their positions. In addition, these relationships can be a starting point for future interdisciplinary collaborations once they move on to their postliminal states. Postdocs could try to collaborate with other postdocs, and if this isn't possible, find other ways to interact with each other, even if this is in a social setting. Having the support of other liminal personae has the potential to help postdocs succeed.

As for advisors and institutions, they can think about the "invisibility" that comes with the liminal state and find ways to provide postdocs with their own, clear status and with support. Some suggestions follow.

First, institutions that are not already doing so should provide access to resources or offices that target postdocs and their needs, such as by establishing a postdoctoral office. Some postdocs in this study expressed that it was not always easy for them to find institutional support or see where they fit in. An office that addresses postdocs' needs and interests can work to ensure that University policies, regulations, and activities all clearly include postdocs as its own classification, in addition to the classifications of faculty, staff, and student which are generally the only ones provided. In addition, because "most institutions appear to have few policies designed for postdocs specifically"1, this office can also begin to develop these policies that will be specifically targeting postdocs and their needs. 
Second, institutions and/or advisors can help postdocs as they learn to see the liminal state as the "rebuilding process" 14 that it can be by providing professional development opportunities, especially those related to career guidance and how to move forward. These types of professional development opportunities can help the liminal personae learn about and develop skills necessary for their "postliminal duties and rights"14. Activities can include seminars on how to apply for industry/academic jobs, or how to learn skills that are necessary for those jobs (for example, how to plan a lesson or how to write a grant). The postdocs in this study expressed that they would like more career guidance. This is consistent with what other studies have found. For example, according to the AAU Report", "career advising and job placement assistance" are areas postdocs identify as needing improvement. In Akerlind's study ${ }^{2}$ on Australian postdocs, postdocs reported a "widespread lack of systematic institutional policies and structures for postdoctoral training or career support." In Helbing, Verhoef, and Wellington's study $^{25}$ on Canadian postdocs, $70 \%$ "of respondents felt strongly that they had not received sufficient counseling on career planning." Institutions can consider creating policies and resources to target postdoc professional development and career support. After all, career guidance should not solely come from the postdocs' advisors ${ }^{12}$.

Another area of professional development the postdocs in this study discussed they needed was teaching. While some postdocs appreciate that they are not required to teach and can focus solely on research, other postdocs strongly desire the opportunity to teach and develop these skills, as they believe this will better prepare them for an academic/teaching career. Advisors can consider taking the time to learn about their postdocs' interests and goals, and providing teaching opportunities for those who are interested in this area. They can also consider doing this in a mentorship role, where they can co-teach with or otherwise help those postdocs who have little experience, yet are interested in learning to teach. As stated in the NAS et al. report ${ }^{4}$, advisors should "provide a postdoctoral experience that is fundamentally educational in nature and advances the postdoc's career," which means including activities, such as teaching, that align with the postdoc's goals.

Finally, the findings presented in this paper can be useful to current doctoral students and faculty who advise them. Advisors can consider informing their doctoral students about what experiences are likely to come with a postdoctoral position, and encouraging them to learn more about the postdoc experience before applying for or accepting one. Doctoral students can consider learning about the liminal state as preparation for what to expect if they decide to pursue a postdoc position. Given the challenges that come from a liminal state, advisors and doctoral students should thoughtfully consider whether a postdoc position is an appropriate next step - if the doctoral student is in a field where a postdoc appointment is not a requirement before moving on to a permanent position, would a postdoc position really be at all beneficial to this student? On the other hand, individuals for whom a postdoc position is a necessary transitional stage in their fields can certainly benefit from knowing more about the experiences that characterize a postdoc position, as this knowledge would better prepare them for this liminal state, both to take advantage of the opportunities and benefits that come with the position, as well as to understand, accept, and possibly ameliorate the challenges that also come with it.

There were some limitations to this study. First, the participants were self-selected, so the experiences of these postdocs may not be entirely representing the science and engineering 
postdoctoral position. Also, much like the graduate student experience, the postdoctoral experience depends on the postdoc's advisor and lab. As such, when reading about these experiences, it is important to remember that they are the result of working with those particular advisors in those particular labs, and may not necessarily be entirely representative of the science and engineering postdoc position.

As stated by Cantwell and Lee ${ }^{21}$, "the postdoctorate warrants further study within the field of higher education." There is little literature on the postdoc experience, especially as compared to existing literature on faculty, graduate student, and undergraduate student experiences. This study sought to contribute to this need by looking at the postdoc experience as a liminal state, and using the theory of liminality to better understand this experience. This study also sought to contribute to the literature by focusing specifically on science and engineering postdocs in the United States. The area of study is important in better understanding the postdoc experience, as different disciplines tend to have different cultures and expectations. This study places these participants in a particular context, so readers can better interpret and compare experiences and recommendations.

There are many possibilities for future research on the postdoc experience. First, because there is a higher proportion of "foreign born" 26 doctorates accepting postdoc positions, future research can continue to look at these postdocs' experiences. Second, future research can continue to look at the postdoctoral experience by discipline and by gender. In this study, when responses from science and engineering postdocs were compared, no significant differences were observed that could be specifically attributed to the respective fields. In addition, the number of female postdocs was much smaller than the number of male postdocs, making it difficult to accurately compare experiences by gender. Third, the postdocs in this study provided certain reasons why they decided to be postdocs (research, training, etc.). Future research should look at postdoc expectations and if and how well these expectations were met in their appointments. As a continuation to this study, future research will focus on getting a more in-depth understanding of the postdoc experience by gender and by disciplines, both within the current institution and across various institutions. By replicating this study at other institutions, it is expected that the generalizability of these results, and consequently, our understanding of the science and engineering postdoctoral experience, will increase.

Although this paper examined the experiences of science and engineering postdocs in one particular university, the overall experiences and the liminal experiences are likely descriptive of the postdoctoral experience in other institutions. In addition, the recommendations provided in this paper should be applicable in and relevant to other institutions that employ postdocs.

\section{Acknowledgements}

The author would like to thank the Leonhard Center for the Enhancement of Engineering Education for providing support for this study. The author would also like to thank Dr. Thomas Litzinger and Dr. Sarah Zappe for their support, feedback, and guidance. 


\section{List of references}

1.Association of American Universities Committee on Postdoctoral Education Report. (1998).

Retrieved August 29, 2012 from http://www.aau.edu/publications/reports.aspx?id=6900

2.Akerlind, G. S. (2005). Postdoctoral Researchers: Roles, Functions and Career Prospects.

Higher Education Research \& Development, 24(1): 21-40.

3.Akerlind. G. S. (2010). Developing as a Researcher Post-PhD. In L. McAlpine \& G. S. Akerlind (Eds.), Becoming an Academic: International Perspectives (45-70). Palgrave Macmillan.

4.National Academy of Sciences, National Academy of Engineering, Institute of Medicine.

(2000). Enhancing the Postdoctoral Experience for Scientists and Engineers. Washington, DC: The National

Academies Press.

5.Davis, G. (2009). Improving the Postdoctoral Experience. In R. B. Freeman \& D. L. Goroff

(eds.). Science and Engineering Careers in the United States. The University of Chicago Press.

6.Castaneda, S. (2009). Who Cares about Postdocs? International Educator. 18(1): 58-59.

7.Horta, H. (2009). Holding a post-doctoral position before becoming a faculty member: does it

bring benefits for the scholarly enterprise? Higher Education, 58: 689-721.

8.Hoffer, T. B., Grigorian, K., \& Hedberg, E. (2008). Postdoc Participation of Science,

Engineering, and Health Doctorate Recipients. InfoBrief, National Science Foundation (NSF 08-307), March 2008, [Electronic Version]. Retrieved May 29, 2013, from

http://www.nsf.gov/statistics/infbrief/nsf08307/nsf08307.pdf

9.Stephan, P., \& Ma, J. (2005). The increased frequency and duration of the postdoctorate career

stage. The American Economic Review, 95(2), 71-75.

10.Fetzer, J. (2005). Postdoctorate in Perpetuity and Other Issues. Analytical and Bioanalytical

Chemistry. 383: 1028-1029.

11. Singer, M. (2004). The Evolution of Postdocs. Science. 306: 232.

12.Scaffidi, A. K. \& Berman, J. E. (2011). A Positive Postdoctoral Experience is Related to Quality Supervision and Career Mentoring, Collaborations, Networking and a Nurturing Research Environment. Higher Education. 62: 685698.

13.Van Gennep, A. (1960). The Rites of Passage. Chicago: The University of Chicago Press.

14.Turner, V. (1977). Variations on a Theme of Liminality. In S. F. Moore \& B. G. Myerhoff

(Eds.). Secular Ritual. Assen, The Netherlands: Van Gorcum. P.36-52.

15.Turner, V. (1967). The Forest of Symbols. London: Cornell University Press.

16.Turner, V. (1974). Dramas, Fields, and Metaphors: Symbolic Action in Human Society.

London: Cornell University Press.

17.Creswell, J. W. (2009). Research Design. California: Sage Publications.

18.Cohen, L., L. Manion, \& K. Morrison. (2011). Research Methods in Education. London and

New York: Routledge.

19.Glesne, C. (1999). Becoming Qualitative Researchers: Addison Wesley Longman.

20.Cook-Sather, A. (2006). Newly Betwixt and Between: Revising Liminality in the Context of a Teacher

Preparation Program. Anthropology and Education Quarterly. 37(2): 110-127.

21.Cantwell, B., \& Lee, J. L. (2010). Unseen Workers in the Academic Factory: Perceptions of

Neoracism Among International Postdocs in the United States and the United Kingdom. Harvard Educational

Review. 80(4): 490-516.

22.Turner, V. (1990). Are There Universals of Performance in Myth, Ritual, and Drama? In R.

Schechner \& W. Appel. By Means of Performance: Intercultural Studies of Theatre and Ritual. Cambridge:

Cambridge University Press. P.8-18.

23.Turner, V. (1969). The Ritual Process: Structure and Anti-Structure. New York: Aldine de

Gruyter.

24.Conroy, J. C. (2004). Betwixt \& Between: The Liminal Imagination, Education and Democracy. New York:

Peter Lang Publishing.

25.Helbing, C. C., Verhoef, M. J., \& Wellington, C. L. (1998). Finding Identity and Voice: A

National Survey of Canadian Postdoctoral Fellows. Research Evaluation, 7(1): 53-60

26.Corley, E. A., \& Sabharwal, M. (2007). Foreign-Born Academic Scientists and Engineers:

Producing More and Getting Less than Their U.S.-Born Peers? Research in Higher Education. 48(8): 909-940. 